\title{
DERECHOS INDÍGENAS, TERRITORIO Y DESARROLLO EN CONTEXTOS TURÍSTICOS V CTUrTI
}

\author{
V Coloquio Internacional de Turismo, Pueblos Indígenas, Comunidades \\ Tradicionales y Afrodescendientes (V CTurTI)
}

\section{Resumen:}

El presente informe contiene la sistematización de los talleres realizados en el marco del V CTurTI llevado a cabo entre el 7 y 9 de noviembre de 2018 en el Campus Villarrica de la Pontificia Universidad Católica de Chile, evento realizado en coorganización con el Centro de Estudios Interculturales e Indígenas, Laboter del Departamento de Geografía IESA de la Universidad Federal de Goiás, LAGERI del Departamento de Antropología de la Universidad de Brasilia, la Facultad de Turismo de la Universidad Nacional de Comahue y el Departamento de Antropología de la Facultad de Filosofía y Letras de la Universidad de Buenos Aires.

Se trataron cuatro ejes temáticos: Turismo y Políticas Públicas, Áreas Protegidas, Extractivismo y Proyectos de Desarrollo, y Procesos de Patrimonialización en relación a pueblos indígenas, comunidades tradicionales y afrodescendientes de Latinoamérica, recogiendo las opiniones de participantes pertenecientes a pueblos indígenas y afrodescendientes, comunidades tradicionales, estudiantes, académicos y representantes de instituciones, que discutieron sobre las principales problemáticas, desarrollando diagnósticos e identificando desafíos y soluciones para afrontarlos. 


\section{Presentación}

El $V$ Coloquio Internacional de "Turismo, Pueblos Indígenas, Comunidades Tradicionales y Afrodescendientes (V CTurTI): Derechos Indígenas, Territorio y Desarrollo en Contextos Turísticos", se orientó a la discusión respecto al fenómeno del turismo en territorios indígenas en América Latina.

El turismo ha sido visto como una oportunidad de desarrollo para los sectores rurales $y$ urbanos indígenas, por lo cual ha sido promovido por diversas instituciones públicas y privadas. Del mismo modo, el turismo se ha considerado como una herramienta alternativa y de convivencia intercultural en cuanto exista autogestión de las actividades por las comunidades involucradas. Sin embargo, considerando el atractivo natural, cultural y económico, el desarrollo del turismo también trae aparejado diversos problemas potenciales como la apropiación de una oferta cultural por agentes no indígenas, la folclorización de la cultura, la inequidad en distribución de ingresos, pérdida de hábitats y de control territorial, generando disputas económicas, territoriales y culturales que tensionan los derechos indígenas internacionales avalados por el Convenio 169 de la Organización Internacional del Trabajo y la Declaración de las Naciones Unidas sobre los Derechos Indígenas y temas que se centra en todas las ediciones del CTurTI en América Latina.

El V CTurTI se realizó en el Campus Villarrica de la Pontifica Universidad Católica de Chile (UC) en la ciudad de Villarrica, Región de la Araucanía, Chile. Esta quinta edición del Coloquio fue coorganizada por el Centro de Estudios Interculturales e Indígenas (CIIR), el
Campus Villarrica de la Pontificia Universidad Católica de Chile, de acuerdo con los dos laboratorios de la sede del CURTI en Brasil desde 2013 - el Laboratorio de Estudios e Investigaciones de las Dinámicas Territoriales (Laboter) en el Instituto de Estudios Socioambientales (IESA) de la Universidad Federal de Goiás (UFG) y el Laboratorio y Grupo de Estudios en Relaciones Interétnicas (LAGERI) del Departamento de Antropología (DAN) de la Universidad de Brasilia (UnB), con colaboracion de la Facultad de Turismo (FaTu) de la Universidad Nacional de Comahue (UNComa) y la Facultad de Filosofía y Letras (FFyL) de la Universidad de Buenos Aires (UBA).

La sistematización de estos resultados es fruto del trabajo colectivo de todos y todas las y los participantes del coloquio provenientes de diversos territorios, pertenencias y ámbitos de vinculación con el turismo.

\section{Antecedentes del CTurTI en Brasil y VI CTurTI en Chile}

El Primer "Colóquio de Turismo em Terras Indígenas: Turismo, Territórios Identitários e Conflitos Interétnicas em Debate (1ํ CTurTI)" se inició a partir de la tesis de doctorado en la interfaz de la Geografía Humana y Antropología Social, titulada - "Os povos indígenas, o turismo e o território: um olhar sobre os Tremembé e os Jenipapo-Kanindé Ceará" - que fue defendida en 2012 por Isis Maria Cunha Lustosa en la UFG/IESA, Brasil. El turismo como fenómeno social y sus implicaciones en los territorios tradicionales, es tema de investigaciones desde 1998 en el IESA, y los estudios sobre el turismo se ampliaron a partir de 2008 en el Laboter/ IESA/UFG. 
El 1ํ CTurTI fue coordinado por las profesoras Isis Maria Cunha Lustosa y Maria Geralda de Almeida en la UFG/IESA/Laboter, junto con algunas universidades brasileñas, a destacar la UnB/DAN/LAGERI con la representación de Dr. Stephen Grant Baines y otras universidades del exterior. En la primera edición del CTurTI fueron priorizados los debates referentes al turismo y las cuestiones territoriales; las políticas indigenistas ante las legislaciones nacionales y los tratados internacionales; los reconocimientos oficiales de los territorios tradicionales y las otras situaciones de derechos tradicionales en Argentina, Colombia y México, justificándolo como evento internacional en Latinoamérica.

Desde el primer CTurTI, las Dra. Almeida y Dra. Lustosa, se propusieron formar una red latinoamericana con participantes (liderazgos indígenas y de otros pueblos y comunidades tradicionales, académicos de las áreas de geografía, antropología y áreas afines al Coloquio), con la presencia de representantes de gobiernos y de organizaciones no gubernamentales que fue fortalecida en el V CTurTI en Chile.

\section{El V Coloquio Internacional de "Turismo,} Pueblos Indígenas, Comunidades Tradicionales y Afrodescendientes (V CTurTI)" es más una instancia de discusión y reflexión respecto al rol del turismo en Latinoamérica, que reúne a académicos, investigadores, representantes de comunidades tradicionales, representantes de instituciones públicas y privadas que promueven el turismo. El V CTurTI mantiene la conformación de la red iniciada en $1^{\circ}$ CturTI y dedicada a estas materias entre los participantes de las distintas versiones del Coloquio, de modo de fortalecer una comprensión intercultural e interdiscipli- naria del turismo en la región. EI CTurTI desde el año 2013, aborda diversos ejes temáticos en sus distintas ediciones, todos asociados al fenómeno turismo en la América Latina.

\section{Objetivos del V CTurTI}

1. Exponer nuevas discusiones sobre el turismo realizado en territorios de pueblos indígenas, afrodescendientes y de comunidades tradicionales, como actividades alternativas en países latinoamericanos contrarias al turismo masivo y otros grandes proyectos de desarrollo que se instalan en los territorios indígenas.

2. Fortalecer la red latinoamericana de organizaciones indígenas y afrodescendientes, académicos, representantes del gobierno y de organizaciones no gubernamentales vinculadas al turismo y promover espacios de intercambio de experiencias sobre el desarrollo del turismo en territorios históricos indígenas, comunidades tradicionales y afrodescendientes en un contexto de progresivo crecimiento del fenómeno turístico en Brasil, Chile, Argentina y otros países de la América Latina.

3. Aportar al debate entre diferentes actores involucrados en el desarrollo turístico vinculado a territorios y organizaciones indígenas y afrodescendientes.

\section{Participantes}

Pueblos indígenas, afrodescendientes, comunidades tradicionales, académicos, investigadores, estudiantes de pre y posgrado, representantes de instituciones públicas y privadas. 


\section{Ejes temáticos del V Coloquio}

\section{Turismo y Políticas Públicas:} pueblos indígenas, comunidades tradicionales y afrodescendientes, visiones críticas desde América Latina

Este eje buscó analizar desde una perspectiva crítica los efectos del turismo en los territorios indígenas, comunidades tradicionales y afrodescendiente, como consecuencia de la aplicación de políticas públicas que permitan construir una mirada comparativa desde América Latina y avanzar en el debate sobre los desafíos futuros relativos a un contexto de reconocimiento de los pueblos indígenas, comunidades tradicionales y afrodescendientes.

\section{Turismo, Pueblos Indígenas y Áreas Protegidas}

Este eje buscó problematizar sobre la relación del turismo, áreas silvestres protegidas y las demandas de control territorial de los pueblos indígenas. Del mismo modo, se espera dialogar en torno a las concepciones de protección de las áreas protegidas como co-manejo, autogestión, entre otros.

\section{Turismo, Extractivismo y Proyectos de Desarrollo: contradicciones y conflictividades en los territorios de pueblos indígenas, comunidades tradicionales y afrodescendientes}

Este eje analizó la relación entre el desarrollo del turismo asociado a los pueblos indígenas, comunidades tradicionales y afrodescendientes bajo parámetros de respeto cultural, ambiental y de derechos indígenas con la presencia de proyectos y actividades extractivistas que afectan estas iniciativas de turismo con acciones tales como conflictos socioambientales, el bienestar de las comunidades locales, el propio turismo y la biodiversidad del territorio.

\section{Turismo y Procesos de Patrimonialización en los Territorios de Pueblos Indígenas y Afrodescendientes}

El eje buscó analizar los procesos de patrimonialización por diferentes agentes y sus disputas asociados al turismo, pueblos indígenas, comunidades tradicionales y afrodescendientes desde una mirada amplia que puede tener efectos positivos o negativos a los procesos de los agentes locales.

\section{Experiencias de Turismo con Pueblos Indígenas y Afrodescendientes en Chile y América Latina: espacios de reivindicación política y territorial}

Este eje buscó compartir diversas experiencias de turismo y prácticas de turismo desde una perspectiva comunitaria, de reivindicación política, cultural e histórica, que desarrollan personas, familias y organizaciones de pueblos originarios, comunidades tradicionales y afrodescendientes. También, plantea poner una discusión sobre los actores involucrados con el turismo y las decisiones sobre los usos del territorio.

Para mayor información: http://www.ciir.cl/ ciir.cl/agenda/v-coloquio-internacional-turismo-pueblos-indigenas-comunidades-tradicio- 
nales-afrodescendientes-v-cturti-derechos-indigenas-territorio-desarrollo-contextos-turisticos/

Como parte de la metodología de trabajo se realizaron Talleres que abordaron los cuatro primeros ejes temáticos del V CTurTI. Se diseñaron pensando en la oportunidad única que ofrecía el carácter del Coloquio, que fue la de poder converger a diversos actores a nivel nacional e internacional, y que están actualmente involucrados en el fenómeno turístico desde la perspectiva de los pueblos indígenas, comunidades tradicionales y afrodescendientes. Desde este punto de vista el desafío fue proponer los cuatro primeros Ejes Temáticos e invitarlos a que, en cada Taller, reunidos de forma voluntaria y aleatoria, pudieran discutir principales problemas, diagnóstico (causas y efectos) y una parte propositiva que atisbara soluciones conjuntas de cada tema.

Posteriormente el Equipo Organizador se dio la tarea de sistematizar toda la información recogida, de manera que pudiera reflejar lo más detalladamente posible las opiniones emergidas y poner en relieve las principales discusiones que están actualmente dándose en el ámbito del turismo y los pueblos indígenas.

\section{Taller Eje Temático 1. Turismo y Políticas} Públicas: pueblos indígenas, comunidades tradicionales y afrodescendientes. Visiones críticas desde América Latina

\subsection{Introducción}

El turismo denominado de intereses especiales, comunitario, indígena, étnico o con algún tipo de apellido que lo diferencia del turismo de masas, se ha posicionado en las últimas décadas en América Latina como una política pública enfocada en la promoción de la inversión en los territorios indígenas, de comunidades tradicionales y afrodescendientes. Estas acciones públicas impactan en la generación de una nueva forma de concebir las prácticas económicas y culturales, así como introducen en estos espacios la pregunta sobre qué experiencias o prácticas culturales son posibles de ofrecer a los turistas que buscan conocer algo que tradicionalmente se ha presentado con carácter de exótico o desconocido.

Esta promoción del turismo no sólo resulta en una acción pública de desarrollo, sino también los pueblos y territorios van haciendo suyo este discurso y potencialmente se transforma en una nueva línea de desarrollo, constituyéndose en una estrategia económica local. Pero también aporta a los procesos de revitalización y/o resistencia cultural, no exenta de cuestionamientos internos en los pueblos y comunidades. Se produce así una apropiación de una política pública por parte de los pueblos indígenas, comunidades tradicionales y afrodescendientes, otorgando un nuevo cariz a una política pensada inicialmente desde el desarrollo económico de los territorios.

\subsection{Principales resultados}

\subsubsection{Desafíos}

a. Se señala que las políticas públicas no sólo deben ser consultivas, sino también que afecten (vinculante) la ejecución de la misma si las comunidades así lo deciden. Esto ha llevado a una continua lucha por actualizar las normas vigentes, lo que ha provocado desconfianza hacia el Estado, que se ve más como una amenaza que como un garante. Se señala que hoy hay un despoblamiento (migración de 
jóvenes) de los pueblos y existe estigmatización de las nuevas generaciones.

b. No existen representantes o autoridades ancestrales validadas por las comunidades, esto provoca que no se puedan transmitir los problemas reales de la comunidad hacia el Estado, donde se puedan asumir puntos de vista desde la cosmovisión de los pueblos indígenas.

c. No hay una buena regularización de los proyectos que involucran a los pueblos originarios y que tienen un impacto sobre la economía. Esto permite que ingresen personas (profesionales) que no tienen pertinencia, que no tienen respeto por la comunidad, ya que desconocen el modo de vida de las comunidades indígenas o conocimiento sobre el territorio. Poseen intereses económicos por sobre todo.

\section{d. Las Mesas Territoriales no son funcionales, lo} que provoca que no existan avances y los líderes se mantienen por periodos muy largos. Se asocia también a malos liderazgos que no transmiten la realidad de las comunidades y muchas veces estos tampoco tienen pertinencia.

e. Las políticas públicas están centralizadas y abordan estrategias de acción sin tomar en cuenta territorios ni especificidades de los diversos pueblos indígenas. Se señala que se da preferencias a los intereses privados y más privilegiados, además de una falta de retroalimentación que no permite una adecuada comunicación entre "occidentales" y pueblos indígenas, pues no se comprenden en lenguajes y formas de entender las realidades. Otra causa, señalan, se enmarca en una discriminación e invisibilización de los pueblos indígenas, que los asumen en una "categoría inferior", sumado a la dependencia económica que tienen muchas comunidades, creando relaciones de paternalismo.
f.Esto provoca la implantación de un modelo único provocando "desequilibrios y desigualdad" para poder desarrollarse, lo que obliga a entrar en lógicas occidentales que no aportan a las comunidades.

g. Se identifica dificultad de diálogo entre universidades, el gobierno y los pueblos indígenas. Mientras que estos siguen empobreciéndose.

h. Uso indiscriminado de los conocimientos de los pueblos indígenas en uso de marcas y registro no autorizado de las mismas. Esto lleva a malas prácticas de los saberes indígenas.

i. Hay una prevalencia de lo urbano por sobre lo rural, generándose proyectos que benefician más a las comunidades urbanas. Existen normativas con respecto a la formalización (sobre todo las autorizaciones sanitarias) de las iniciativas turísticas que se aplican igual modo tanto para el espacio urbano como para el rural. Lo que ha provocado deserción y abandono de la actividad turística.

j. Las políticas internas muchas veces segregan y mutilan a las comunidades indígenas, y crean conflictos por ejemplo entre las Juntas de vecinos y las comunidades indígenas, provocando divisiones en los territorios.

k. Se plantea que no hay una claridad sobre lo que se entiende por turismo indígena, ya que por un lado está el emblema del desarrollo y por otro la cultura del buen vivir. En esta disyuntiva, el Estado no escucha las demandas de los pueblos indígenas, por ejemplo, se ejecutan proyectos desde el gobierno, a través de organismos asesores, que desarrollan planes de capacitación, estudios, etc., que son reiterativos sin avances sobresalientes. Esto ha llevado a que dirigentes de comunidades indígenas se 
vean sobrepasados con reuniones, capacitaciones y eventos que no asumen las opiniones, por tanto, se produce una mala inversión de los recursos fiscales.

I. Además, hay comunidades indígenas que no están del todo organizadas, no hay una contraparte que actúe como articulado para poder dirigirse al Estado. Cuando se reciben recursos, hay dificultades respecto a su administración, ya que las organizaciones no cuentan con recursos técnicos para hacerse cargo.

m. Se percibe al turismo como una actividad extractivista (en la situación de Chile) ya que usa mecanismos de la "tradición occidental", por ello muchas veces las comunidades no entienden esos lenguajes. Por eso hay comunidades que observan al turismo como una amenaza, y efectivamente, señalan, hay territorios donde el turismo ha tenido resultados desastrosos.

n. Por eso hay comunidades que observan al turismo como una amenaza. Hay lugares, como nuestro territorio, en las que los resultados del turismo han sido desastrosos.

\subsubsection{Propuestas}

a. Se plantea que las normas deben respetar la cultura de los pueblos originarios, con especificaciones técnicas pertinentes (problemas referidos a normativas sanitarias).

b. Se plantea que se deben integrar y validar autoridades ancestrales legitimadas, en el diálogo con el Estado, sobre decisiones que los afecten.

c. Se plantea que, al ingresar un proyecto, este debe tener una carta de autorización y respaldo de las autoridades tradicionales.
Además, las comunidades indígenas deben tener completo conocimiento de los proyectos antes de que se ejecuten.

d. Que las mesas territoriales puedan tener recambio de líderes, sobre todo, que puedan estar las nuevas generaciones y apoyar el poblamiento de las comunidades.

e. Se plantea la idea de cuotas a representantes indígenas en el parlamento. Además, se debe apuntar a la descentralización de las políticas públicas y que las autoridades regionales dialoguen con las comunidades. También se deberían incorporar y reconocer los tratados internacionales para buscar igualdad. Crear escuelas de liderazgos entre indígenas y no indígenas, para aseguir aprendiendo y desarrollándose.

f. Los pueblos indígenas deben ser protagonistas de las políticas públicas que los afecten.

g. Se plantea que debiese existir una Sociedad de Derecho de Autor indígena y que la gente pague por el usufructo de las mismas.

h. Se plantea que debe haber acciones públicas que mejoren la conectividad de las zonas urbanas. Además, se deben fortalecer los liderazgos, creación de auto gobernanza turística junto a una coordinación institucional de instrumentos de apoyo por parte de los gobiernos. Se exige una mayor coordinación interinstitucional.

i. Se plantea "generar marca o sello propio de turismo indígena reconociendo las particularidades de cada pueblo indígena y contar con protocolo para certificar".

j. También se plantea fortalecer los gremios 
a nivel local, regional y nacional, para lograr la articulación y contar desde los gremios una contraparte que articule con los distintos actores.

k. Un funcionario público de Chile señala que en el 'turismo indígena' (como es denominada la actividad en Chile), se pueden hacer categorías y certificar en distintos niveles. Se plantea desarrollar un modelo que sirva de diagnóstico para el reconocimiento, ver quiénes están, de emprendimientos indígena y su certificación. Señala que tiene claro que es difícil implementar ciertos modelos. Esta es una idea que está en fase de diagnóstico, es un modelo canadiense que se tiene que adaptar a la realidad chilena, por lo que primero se hará un piloto en un territorio aún por definir.

I. Un líder del pueblo mapuche, plantea que lo de la calidad es un tema de importancia. Señala que el turismo indígena como algo nuevo, por lo que estas instancias se deben aprovechar para ponerse de acuerdo. Bajo esta premisa es que se pensó en crear una organización a nivel nacional de turismo indígena para formular soluciones a largo plazo. Aclara, que hay que distinguir que no hay fórmulas únicas, porque todos los pueblos indígenas son distintos.

m. Se plantea que el Estado debe dar prioridad a las comunidades encargadas de resguardar sus territorios.

n. Se señala que es necesario poder integrar las lenguas originarias a los distintos territorios como una política pública "real", debe ser enseñada por hablantes de las comunidades.

o. Se señala que se debe exigir que los estudios o investigaciones científicas deben citar a las personas de las comunidades indígenas que participaron y el deber de los encargados de los estudios, de presentar los resultados a las comunidades primero que todo.

\section{Taller Eje Temático 2. Turismo, Pueblos Indígenas y Áreas Protegidas}

\subsection{Introducción}

El Estado chileno, durante el siglo $X X$, ha determinado que algunas zonas del país tienen un valor público que es necesario proteger para preservar algunas características, especialmente ambientales. Estas zonas se definen como áreas protegidas ${ }^{1}$ y están sujetas a diferentes normativas que indican $u$ obligan distintos tipos de uso del territorio. Sine qua non, estas áreas protegidas poseen un alto valor para el ecosistema como espacios de conservación natural de especies y biodiversidad, así como también de paisaje. No obstante, este paisaje normado suele invisibilizar el uso y ocupación humana que ha contribuido a su modelación, marginando la influencia de prácticas, generalmente tradicionales, en su caracterización, como se informa para el pueblo Aymara y Mapuche. Salvo algunas excepciones y/o distinciones-dependiendo de la región y dinámicas en las relaciones público-privadas/formales e informales; administrativas, étnicas y socioproductivas, entre otros aspectos-, en las que estas áreas protegidas fundan su operatividad en relación con especificidades territoriales como, por ejemplo, las áreas de desarrollo indígena como es el caso de San Pedro de Atacama.

En consideración con estas especificidades y características, muchos de estos lugares, se perfilan como núcleos de atracción turística por su riqueza natural y cultural por ofrecer al 
visitante una experiencia única y diferenciada de su actividad cotidiana.

En correspondencia con lo anterior, estas áreas protegidas, públicas o privadas, hacen parte de los territorios históricos de los pueblos indígenas, quienes tienen asociados prácticas culturales, sociales y económicas, y forman parte de sus demandas políticas actuales. En este marco, el territorio protegido pasa a tener sentidos diversos para quienes lo habitan, lo que define diferentes prácticas y relaciones, generando tensiones entre los elementos simbólicos, económicos y de conservación, sólo por nombrar algunos.

A partir de esta reflexión uno de los elementos relevantes que está al centro de estas cuestiones es: ¿cuál es la influencia que tienen las comunidades sobre estos territorios?, territorios que han sido declarados espacios de protección natural al interior de territorios tradicionalmente ocupados por comunidades indígenas y que en muchos casos están en procesos de regularización o en conflicto por su pertenencia. Razón por la cual, las comunidades y grupos originarios sin adscripción, como norma, no tienen influencia en la toma de decisiones sobre sus propios territorios.

Esto también evidencia el tipo de colonización que se produce a partir del desarrollo de un modelo productivo basado en la ley que separa la cultura de la naturaleza, circunscribiendo la relación del espacio en este marco de naturaleza, que propende solo a un turismo de paisaje y no de prácticas humanas que configuran paisaje cultural. En estos términos, desde las comunidades indígenas surge la pregunta: ¿sólo es el paisaje lo que se debe conservar?
Responder esta pregunta resulta complejo ante las dinámicas de relaciones formales que el Estado construye con las comunidades, fundado en discursos de reconocimiento $y$ colaboración a través del mecanismo de co-administración de reservas y parques nacionales, que remite a elementos puntuales tales como el corte de la entrada a la reserva o parque y la gestión administrativa, reduciendo el alcance de las comunidades en la toma de decisiones, manteniendo restricciones estandarizadas por medio de las normas que aplican en estas áreas y que marginan prácticas ancestrales de manejo cultural. Al no integrarlas, las comunidades indígenas observan impactos en el ecosistema que modelan un paisaje que invisibiliza la influencia cultural predecesora al Estado. Influencia que se considera importante visibilizar y conservar. "Toda vez que no nos hemos preguntado qué es lo que queremos conservar".

Por otro lado, el Estado ha otorgado concesiones como estrategias de mercado para hacer producir estas áreas protegidas que, a la postre, conforman áreas de exclusión de prácticas culturales que, en consecuencia, no se conservan. Ante este escenario, la reflexión de las comunidades se torna crítica y pone en relieve el conflicto situado en el marco de las demandas territoriales pendientes. De las que se excluyen las áreas protegidas.

\subsection{Principales resultados}

\subsubsection{Desafíos}

a. Disputa por la tenencia de la tierra. En este punto se señala que no se reconocen las tierras de las comunidades y que tiene como correlato la apropiación de las tierras indígenas por parte de los Estados chileno y argentino, mediante 
las denominadas "Pacificación de la Araucanía" y "Campaña del desierto", respectivamente. Se ha visto una manifiesta privatización de los territorios ancestrales, siendo estos administrados por el Estado o por privados. Se señala, además, que el gobierno pone demasiados obstáculos para la regularización de las tierras indígenas, no así para las empresas. Así también, como la existencia de problemas legales, títulos de dominios no reconocidos y las disposiciones legales que regulan las áreas protegidas. Todo esto ha llevado a señalar que existe un aparente antagonismo entre pueblos indígenas y el Estado, cuando este se apropia fiscalmente de los territorios.

b. Dificultades para el ejercicio de la autodeterminación territorial. Se plantea que el Estado no reconoce el derecho a la autodeterminación de las comunidades, es así que las comunidades no pueden ejercer su derecho a elegir su forma de desarrollo y su vida. Se indica que las leyes son contradictorias y que no hay respeto por los tratados internacionales. Esto ha llevado a un escaso control de las comunidades sobre sus territorios.

c. Restricciones burocráticas e ideológicas para el manejo territorial desde el Estado hacia las comunidades indígenas Se señala que existe una "invisibilización" de las capacidades de las comunidades indígenas en relación al manejo, protección y uso territorial, así como una indiferencia ante los conocimientos de los pueblos ancestrales. Se plantea que el Estado pide estándares inviables para las comunidades.

d. Traslado de concepciones territoriales occidentales a los espacios de áreas protegidas. La división arbitraria de la naturaleza y la cultura por parte del Estado, que no cree en la protección de la naturaleza con personas en su interior. Ya que no se hacen distinciones de los distintos modos de entender la naturaleza en relación a la visión de las diversas culturas. Es así que se asume un único concepto de Territorio de área protegida para diversas ontologías respecto al territorio por parte de los pueblos indígenas. Se señala que hay concepciones mercantiles de la naturaleza, en este sentido el sistema capitalista privilegia lo que se puede "vender". Se cree que el turismo indígena va a habitar un espacio territorial igual como lo haría un turismo realizado por alguien no indígena, por tanto, se desestima los modos distintos de habitar, por ejemplo, uno comunitario.

e. Problemas internos que impiden ponerse de acuerdo con el Estado y/o privados. Se señala una falta de organización y empoderamiento de las comunidades indígenas. Se plantea que hay dificultades internas de las mismas, como problemas de comunicación y de articulación que debilitan la capacidad de diálogo con los demás actores.

f. Escasa influencia de las comunidades sobre sus territorios. Las comunidades no tienen influencia en la toma de decisiones sobre sus propios territorios. Se señala que esto se debe al desgaste del territorio, la capacidad de carga y la poca información que entrega el gobierno. Esto ha causado que los jóvenes indígenas migren a las grandes urbes porque no tienen identidad territorial, ni oportunidades laborales. Otra causa, se plantea a la colonización y relativización de la colonización territorial que propende solo a un turismo de paisaje y no de prácticas humanas.

g. Pérdida de identidad territorial, costumbres y tradición en el uso del territorio. Se señala que 
actualmente se vive una pérdida de identidad cultural en relación a sus territorios y acceso territorial. Dejando de lado las prácticas ancestrales, siendo un motivo la llegada de otras religiones. Se plantea la pérdida de la lengua y la vinculación con la naturaleza. Se suman a estos factores, el empobrecimiento y discriminación en las comunidades.

h. Dificultades para definir un desarrollo territorial desde las comunidades indígenas. Se señala que hay una fragmentación y un aislamiento de las comunidades indígenas. Así como dificultades para desarrollarse y desarrollar su vida en su territorio, que amenaza los modos de vida tradicionales.

i. Obstrucción administrativa y simbólica para la administración indígena. Se plantea que hoy más bien existe co-administración "de papel", ficticios, no legítimos, entre comunidades y el Estado. Se señala que hay una sobre burocratización para el manejo de esta vinculación. Finalmente se señala que hay una desconfianza hacia las comunidades sobre la administración de áreas protegidas, no así para la concesión de grandes empresas (privados).

\subsubsection{Propuestas}

a. Legislar con respeto y en bien de la comunidad. Devolución de los territorios ancestrales. Se plantea que se debe impulsar los territorios indígenas como zonas protegidas, reacondicionadas por el Estado. Se señala que el gobierno central debe devolver los territorios para que vuelvan a ser autónomos. Así como la entrega, por parte del Estado, de los territorios donde actualmente existen los parques nacionales a quienes tienen derechos ancestrales sobre ellos. b. Fortalecer derechos indígenas sobre el territorio para generar más control. Se plantea que se deben desarrollar proyectos estratégicos que paulatinamente establezcan el control total del territorio por parte de las comunidades indígenas que viven en él. Se debe pasar de la figura del co-manejo, al control de las áreas protegidas y el territorio ancestral por las comunidades indígenas locales. Debieran existir territorios indígenas de conservación con formas de hacer gobernanza autónoma sobre el territorio desde las comunidades, vinculado a una apertura y el derecho de las comunidades sobre los territorios. Se plantea realizar un catastro arqueológico para validar el territorio indígena e impedir el uso empresarial (mineras, hidroeléctricas, inmobiliarias etc.). Demostrar, recuperar y visibilizar la identidad indígena, presente en el territorio por medio de actividades y ceremonias ancestrales.

c. Educación "Ambiental" con métodos de pertinencia cultural en cada territorio. Se señala que las políticas públicas deben estar generadas a partir de voluntades en términos legales de forma no asistencialista y/o paternalistas. Debe haber una revitalización de la co-administración y sus implicancias. Que los sectores público y privado consideren a los emprendedores con estándares del contexto indígena, y que estos sean vinculantes.

d. Dar valor al conocimiento indígena de conservación tradicional o "Küme Mongen o Buen vivir". Se plantea que la Educación debe recrearse con métodos contextualizados en cada territorio. Se asume la importancia de que exista mejor y mayor Educación ambiental. Aquí se plantea que debe haber una articulación y respeto hacia los guías locales en la valoración de su conocimiento. 
e. Incorporación de idioma ancestral y significados indígenas para la incorporación de concepciones de la naturaleza desde la cultura indígena local. Reconocimiento del idioma ancestral, así como la urgente integración como asignatura en el currículo escolar. Se asume la construcción de otras subjetividades sobre los pueblos. Se plantea que debieran existir nuevas conceptualizaciones del territorio y de las áreas protegidas.

f. Reforzar temas espirituales (Newen).

\section{Taller Eje Temático 3. Turismo, Extracti- vismo y Proyectos de Desarrollo: contradicciones y conflictividades en los territorios de pueblos indígenas, comuni- dades tradicionales y afrodescendientes}

\subsection{Introducción}

El turismo ha sido impulsado como una herramienta que puede desempeñar un papel relevante en el desarrollo de las comunidades indígenas, aunque estos beneficios y costos son desigualmente compartidos dentro de los territorios generando un debate acerca de si la mercantilización de la cultura y territorio ayuda o no a los procesos políticos de autonomía local.

Las incipientes consecuencias y sus efectos ya se dejan ver sobre el territorio, debido a que inciden sobre los medios de vida e instituciones sociales y políticas a diferentes niveles sociales y de gobierno. Los pueblos indígenas, comunidades tradicionales y afrodescendientes han desarrollado formas de turismo que incorporan demandas de reconocimiento, de reivindicaciones históricas, territoriales y culturales. Para estos grupos, el empoderamiento local y el desarrollo a través de iniciativas turísticas ha sido un vehículo para prevenir la apropiación del territorio, recursos naturales y cultura por parte de agentes externos.

La expansión de actividades extractivas y proyectos públicos ha generado conflictos $y$ contradicciones en la relación de estos grupos locales con el Estado, empresas extractivas y otros actores sociales. Por una parte, la globalización económica ha aumentado la presión sobre el territorio y generado conflictos por el control de los recursos naturales, patrimonio y cultura, modificando no sólo la relación entre pueblos indígenas, comunidades tradicionales $y$ afrodescendientes, y los actores productivos y el Estado, sino que también al interior de las familias que son parte de los espacios en disputa. Sin embargo, algunas comunidades indígenas han logrado desarrollar complejas redes con ONGs, políticos locales, regionales y nacionales, otras actividades productivas y organismos del Estado con el propósito de defender el territorio y patrimonio local.

Los procesos de turistificación ${ }^{2}$ del territorio generan valor económico y simbólico, posibilitando no sólo negociaciones económicas y políticas con el Estado y el sector extractivo, sino que también ganancias político-económicas para las comunidades locales y desde esta perspectiva de alguna manera generan el progreso prometido. Sin embargo, el desarrollo que se produce se enmarca en un modelo capitalista el que no permite la incorporación de elementos culturales propios como concepciones y relaciones con la naturaleza. Estos entran en conflicto constantemente, a partir de estas contradicciones se pone a prueba la capacidad de las comunidades indígenas de ejercer su derecho a la autodeterminación, pero que se tensionan con las políticas públicas de 
incentivo productivo que impulsan la gestión turística local y la capacidad de las comunidades de mantener su tradición y cultura sin mercantilizarla.

\subsection{Principales resultados}

\subsubsection{Desafíos}

a. Falta de cumplimiento de las leyes nacionales e internacionales. Se señala que existe una falta de cumplimiento de las leyes por parte del Estado, tanto de las leyes nacionales como las leyes que firma el Estado en un contexto internacional. A su vez, las consultas indígenas no son tomadas en cuenta de manera vinculante por lo que se realizan solo para cumplir la norma. Por ello no se aprovecha de buena forma o en totalidad las herramientas como el Convenio 169, Ley lafkenche, que serían usados mejor si fuesen conocidas por todos.

b. No se asigna el valor debido a los pueblos originarios. Proyectos de desarrollo son muy distintos a los proyectos de vida de las comunidades. Se señala que no existe una concientización del valor de los pueblos originarios por parte de los Estados. Por tanto, no existe una valorización por parte del sistema hacia las comunidades indígenas.

c. El único modelo que se considera es el extractivista aunque en la práctica esté en constante controversia y conflicto con otros modelos. Se plantea que el único modelo que se considera en la toma de decisiones y el que prevalece es el extractivista. Se señala que este modelo no concibe la naturaleza como un ser digno de respeto, sino como un bien explotable y exportable hacia afuera por las trasnacionales.

d. Excesiva burocratización e ineficiente gestión del gobierno. Se señala que existe una descoordinación entre las municipalidades y los organismos públicos, esto hace que se pierda la confianza en estos organismos.

e. Estrategia público-privada para imponer un modelo extractivista homogeneizante. Esto tiene como consecuencia: usurpación de tierras, se ve a las personas como consumidores, desinformación, incentivar la desorganización, lucro, etc. Se señala que la planificación del territorio chileno es para el uso y el interés de lucro de las grandes empresas. Se plantea que hay una desorganización de los pueblos originarios y una ciudadanía desinformada, una falta de educación por parte de ella, eso permite que se los vea como consumidores. Así se generan cooptaciones de liderazgos porque así es más fácil de convencer a las comunidades. Existe una invasión del territorio y la normalización legislativa con respecto a esto. Se señala que se promueve un sistema económico neoliberal y un incentivo forestal por parte del Estado y los subsidios.

f. Comunidades y naturaleza avasalladas por el Estado y el modelo extractivista. Produce división de la población afectada. Impide articulaciones propias y autónomas de "visión de futuro desde las comunidades". Se señala que las comunidades se ven avasalladas por el Estado. A su vez hay un deterioro ambiental y degradación de suelos y mares, de esta forma se fomenta el aumento del cambio climático. Se plantea que hay un aumento de la pobreza al no contar con tierras y espacios para desarrollarse productivamente, culturalmente y de educación por lo que se genera desarraigo cultural y cambios de hábitos. Se plantea que hay una falta de reconocimiento de los territorios de los pueblos originarios. Así, el Estado valora lo 
económico antes que lo cultural y ecológico por lo que no hace consultas antes de repartir las tierras o dejarle el camino fácil a una empresa trasnacional. Como efectos, se señala la división de la población que habita el territorio afectado, el impacto visual de la explotación del territorio, el daño agroecológico y la pérdida de identidad.

g. Desconocimiento de leyes y convenios que puedan proteger técnicamente a los pueblos originarios. Se señala que no hay una educación o no se conocen las bases de los convenios internacionales dirigidos a los pueblos indígenas. Se señala respecto a las normativas que protegen a los pueblos indígenas, como necesario identificar por qué estas no se están cumpliendo.

h. Imposición de parámetros urbanos (sanitarios y administrativos) en espacios gestionados tradicionalmente. Es difícil establecer el turismo comunitario, a pesar de que está presente en el Convenio 169, ya que los servicios sanitarios rompen el esquema de la demostración de lo tradicional, por lo que se convierte en un problema.

i. No se respetan a los pueblos originarios, en tanto no se les reconoce su territorio y se genera una desvinculación con la tierra. Se impide el ejercicio de la autodeterminación cuando no se les da el debido reconocimiento y se deslegitiman los saberes ancestrales. Se señala que los pueblos indígenas se ven como un problema y no como un valor. Esto ha provocado procesos de violencia y corrupción por parte de los privados a través de compras a individuos o familias. A su vez, de una pérdida de la cultura y una cultura desterritorializada, lo que causa una debilitación y desarticulación de los pueblos originarios.
j.Pérdida de espacios, territorio y naturaleza por la instalación de proyectos extractivistas. Se señala que hay una pérdida de la biodiversidad nativa, degradación de los suelos, pérdida de territorios ancestrales por la usurpación. Esto, plantean, produce una pérdida de los territorios que se hace efectiva cuando llega la industria y se da un fenómeno centrífugo.

\subsubsection{Propuestas}

a. Inclusión de las comunidades en los procesos de elaboración (diseño-desarrollo y cierre) de los proyectos que se quieren instalar en sus territorios para su conocimiento y evaluación. Dentro de los proyectos es importante destacar que, si bien hay algunos que buscan fortalecer a las comunidades, el ideal sería que estos vengan al momento de ingresar a una licitación con el respaldo de las comunidades. No se pueden adjudicar proyectos de personas que lleguen a la comunidad sin que las comunidades participen de la elaboración de los proyectos, ya que son estas las que saben que requieren y necesitan.

b. Valoración de los pueblos originarios, como sus formas de entender, de ser y hacer. Debe haber una valoración de los pueblos originarios para que se respeten. Además de difundir y considerar los derechos de los pueblos originarios.

c. Incorporar la educación ambiental con contenido cultural transversalmente en espacios públicos y privados. Se señala importante fomentar las actividades de educación ambiental, así como reeducar para que se valoricen los conocimientos ancestrales. Además de integrar a las comunidades en la base de la cosmovisión de los pueblos indígenas. 
d. Reestructuración constitucional para generar mayor participación indígena y restitución territorial. Se plantea la restitución de los territorios despojados. Además, se debiera incluir en las cámaras de diputados y senadores al menos a un representante de cada pueblo originario. Se plantea una reestructuración constitucional con participación indígena.

e. Utilizar el turismo (indígena, comunitario, cultural, sostenible) como una herramienta para construcción de nuevos futuros. Se plantea ver el turismo como una herramienta para educar a las nuevas generaciones y a los visitantes, revalorizar a quienes no se sienten parte de un pueblo indígena. Se reflexiona que es una herramienta económica y ecológica porque se ejerce sobre un territorio de manera comunitaria por los mismos pobladores por lo que el ambiente se va a ver beneficiada. Se plantea como una actividad que no explota los recursos naturales, además como una herramienta de fortalecimiento territorial porque gracias a este las personas se sentirían parte del territorio, tienen la oportunidad de relatar su cultura. Así el territorio podría surgir no de una manera extractivista, sino que sustentable y sostenible.

f. Potenciar la sinergia entre el conocimiento y capacidades colectivas. Trabajar en red. Kelluwün (reciprocidad), una red local. Se plantea empoderar a la gente de los territorios. Fomentar las capacidades en materiales de derechos en los pueblos indígenas en materias medioambientales. Una correcta organización eco territorial consecuente con la eco cosmovisión de las comunidades ancestrales, que la ciudadanía genere el cambio.

\section{Taller Eje Temático 4. Turismo y Procesos de Patrimonialización en los Territorios de Pueblos indígenas y Afrodescendientes}

\subsection{Introducción}

Los procesos de re-emergencia, fortalecimiento de la identidad y las demandas indígenas y afrodescendientes han sido impactados de diversas maneras por el turismo, lo que ha incidido, además, en el surgimiento de procesos de patrimonialización en que elementos culturales que se consideran propios están siendo puestos a disposición para el fortalecimiento de las iniciativas de turismo en sus territorios. Lo anterior ha implicado una demanda por el control patrimonial lo que ha traído conflictos con diversos actores y disputas por el patrimonio, como también la puesta en escena de lo propio con fines de exhibición, que han generado disputas al interior de las comunidades. En este eje se convoca a reflexionar sobre estos procesos, acerca de cómo están operando estos mecanismos de patrimonialización, el impacto que tienen en las comunidades que ejecutan iniciativas turísticas y la incidencia en la revalorización identitarias, las relaciones interétnicas y en la folclorización o banalización de aquello que se considera indígena en nuestra sociedad.

Dentro de la complejidad que se asume en relación al patrimonio y su uso turístico, se entrevé el uso y aprovechamiento del patrimonio indígena (empleo de nombres, gastronomía, etc.) por iniciativas que son administradas por no indígenas, desencadenando disputas relacionadas a su pertinencia cultural. 


\subsection{Principales resultados}

\subsubsection{Desafíos}

a. Se señala que hay un desconocimiento de la cultura y lo que se pone en valor, eso hace que a los emprendedores indígenas les cueste ponerse de acuerdo y finalmente se termina mostrando el "desconocimiento de nuestra historia". Se plantea que el turista viene a ver al "mapuche con sus vestimentas", es así como se vende el turismo afuera. Esto ha causado conflictos, como el rompimiento del capital social, hay problemas de "transculturización y mercantilización del producto turístico".

b. Se señala que conceptos como patrimonio, no son propios de los pueblos originarios y que muchas veces son impuestos, por lo que sienten que deben adaptarse a ellas. Plantean que este concepto viene "estereotipado con la idea de museo... es un relato de lo que fue, de lo que hubo y no da cuenta de los sujetos que aún existen".

c. También señalan que hay una pérdida de la cultura y que esta debe ponerse en valor. Esto ha causado, señalan, que hay nuevas herramientas que ellos no manejan y que cuesta incorporarlo al desarrollo del turismo y cómo esto se enfrenta.

d. Señalan que es el mercado quien define las condiciones de la oferta en una relación asimétrica que termina obligando a "que se coloquen las plumas en la cabeza". Hay un estereotipo, de un "mapuche auténtico, foto de museo", que no da cuenta de las realidades actuales de las comunidades, que podrían ser muy atractivas para otros segmentos de turismo.

e. Esto causa, señalan, que el patrimonio "opera como la búsqueda de lo auténtico. Termina siendo una suerte de engaño al turista, "porque ese auténtico mapuche es una figura de museo". Esto provoca, que las comunidades, se tengan que diferenciar y "disfrazarse".

\subsubsection{Propuestas}

a. Se plantea que todos deben asumir sus roles en la sociedad, ya sean como profesionales, funcionarios públicos, y no permitir la folclorización. Es así que conocer la historia es importante para poder mostrarla.

b. Además de pensar en una planificación del turismo en conjunto, escribir sus lineamientos. Que las universidades asuman problemas para dar soluciones y la información recogida no quede solo en el ámbito académico. Por ello, se plantea que las universidades debiesen crear redes que ofrezcan capacitaciones a líderes en el ámbito del turismo.

c. Señalan que deben apropiarse de la palabra patrimonio, no solo de una forma conceptual, sino también de su significado. Plantean que el turismo que tiene que ver con comunidades o pueblos originarios, debe tener organismos 0 un operador turístico que produzca filtros para no traer a cualquier turista, sino quienes estén informados para que pueda entender y conocer de estos conocimientos.

d. Se plantea que debe haber un diálogo entre mundo público y el privado, además de la academia para desarrollar el turismo "como nosotros lo queremos", para no asumir fórmulas impuestas por el sector público.

e. Plantean que son las comunidades quienes deben definir lo que es patrimonio, lo que "es sagrado y lo que es profano". Esto lo han hecho ya algunas comunidades, justamente por una invasión del turismo, en que han visto 
sobrepasada su propia intimidad y necesitaron poner límites.

f. Señalan que es importante reconocer y vincularse con segmentos del mercado que valoren la manera de hacer cultura, la forma de ver el patrimonio. Ser selectivos en este sentido.

g. Además, les parece importante desarrollar modelos de gestión que respeten la cultura, que no necesariamente sean una empresa, que reconozcan las dinámicas de la comunidad.

h. Se plantean dos posiciones interesantes del turismo mapuche en Chile: Una mujer mapuche dedicada al turismo mapuche señala que "como mujer mapuche hacemos un turismo para defender nuestra Mapu Ñuke (madre tierra), nuestro lof (comunidad indígena), ese es nuestro rol, sin nuestro territorio nosotros no estaríamos aquí". Agrega que fue distinguida en su labor turística este año, señala que "partí haciendo turismo con tortillas de rescoldo, pero vengo hace años luchando por nuestras tierras. Siempre hicimos trafkintu (espacio de intercambio de productos sin dinero de por medio), me fui a estudiar energía solar fuera de Chile a India". Plantea que allá "se sintió wingka (persona no mapuche) dentro de los indios, siendo mapuche. Ahora entiendo cuando muchos pueblos indígenas son discriminados. Yo allá me sentía discriminada como mujer. Allá las mujeres son muy discriminadas, nosotros vivimos en un país libre, eso me llegó como la fortaleza que tenemos como mapuche, de salir de mi puerta y encontrarme con el bosque, de tomar el agua pura. La debilidad que tenemos es no hacer visible nuestros instrumentos internacionales con respecto al turismo, el turismo es un derecho, no es para generar lucas (dinero), sino que es un derecho propio, es la reivindicación de nuestras vidas, para el futuro de nuestro pueblo".
Un hombre mapuche, dedicado al turismo también plantea que ellos están trabajando en su territorio, una propuesta de turismo originario donde se trabaja en conjunto con los consejos, en comunidades. Plantea que "con todo nuestro pueblo estamos sacando un modelo de turismo. En estos momentos ha sido una labor comunitaria, no ha intervenido el Estado ni consultoras. Todo ha nacido del pueblo. Ha sido un trabajo arduo... nuestra intención es vender directamente nuestro producto al extranjero sin pasar por agencias. Sacamos un sello de calidad turística y nos falta el sello de sustentabilidad para vender directamente". Esta idea nace, señala porque en "nuestro territorio hay salmoneras y forestales. Las salmoneras hacen que la gente abandone las comunidades y se vayan a trabajar con ellos: existe un daño social y ambiental. Estamos tratando de dar una iniciativa desde nuestro pueblo, que es de conservación".

i. Se plantea la necesidad de llegar a acuerdo sobre qué se entenderá por patrimonio para los pueblos indígenas. Se plantea que se puede hablar de "itrofill mongen" (palabra en mapundugun, que quiere decir biodiversidad), ya que es "una idea circular de lo sagrado, más que de la apropiación, que es patrimonio. "Uno no puede ser dueño de lo que se es parte". Señalan que como no se reflexiona, se definen categorías ajenas y con eso construyen políticas públicas.

\section{Reflexiones finales}

Los talleres participativos en el marco del V CTurTI, lograron generar un insumo de interés respecto a temáticas contingentes relacionadas a pueblos indígenas, comunidades tradicionales y afrodescendientes, desde sus propios protagonistas. 
Muchas de las reflexiones fueron trasversales a los 4 ejes temáticos abordados en los talleres. El reconocimiento y demanda por el territorio se configuró como una cuestión fundamental y transversal a todas estas temáticas, de lo que se desprendían dificultades particulares. Destacaron planteamientos en torno a los procesos de asistencialismo y dependencia estatal, así como la imposición de un modelo neoliberal y extractivista que se traduce en políticas públicas de facilitación de acceso de privados no indígenas a los territorios, para el desarrollo de proyectos de inversión que afectan directamente a las comunidades en términos culturales, identitarios y de uso. Similar a lo que ocurre en áreas protegidas públicas y privadas que niegan acceso e invisibilizan la pertinencia de grupos humanos a las mismas, previa a su creación, desvalorando e invisibilizando el potencial indígena para su manejo. Por lo que se vuelve necesario la integración de los pueblos indígenas, comunidades tradicionales y afrodescendientes en los procesos de elaboración de políticas públicas que respeten la legislación y convenios nacionales e internacionales que protegen sus derechos, en conjunto con la consideración de las implicancias socio ecológicas de las decisiones políticas, en miras al "buen vivir" o Küme mogen, en tiempos de cambio climático y de peligro de nuestro medio ambiente. Finalmente, se expresó la necesidad de una re-valorización y un conocimiento profundo de la cultura para la creación de productos turísticos, sin caer en su folclorización, ni mercantilización, otorgando un producto y servicio al turista que dé cuenta de la realidad actual vivida por las comunidades, educando en sus saberes.

\section{Notas}

\footnotetext{
1 "Un espacio geográfico claramente definido, reconocido, dedicado y gestionado, mediante medios legales $u$ otros tipos de medios eficaces para conseguir la conservación a largo plazo de la naturaleza y de sus servicios ecosistémicos y sus valores culturales asociados" Convenio sobre Diversidad Biológica 1994. En: Dudley, N. (Editor) (2008). Directrices para la aplicación de las categorías de gestión de áreas protegidas. Gland, Suiza: UICN. $x+96 \mathrm{pp}$.

2 "A turistificação [...] tem subvertido a utilização tradicional dos lugares [...]. Pouco a pouco, os equipamentos turísticos instalam-se e consolidam-se os enclaves criados En: Maria Geralda de Almeida, 1997, p. 31, Turistificação - os novos atores e imagens do litoral cearense apud Lustosa, Isis Maria Cunha, Os povos indígenas, o
}

turismo e o território: um olhar sobre os Tremembé e os JenipapoKanindé do Ceará, 2012, Tese (Doutorado) - Universidade Federal de Goiás, Instituto de Estudos Socioambientais.

El concepto de turistificación busca dar cuenta del proceso en que los territorios indígenas cambian su vocación económica y de desarrollo hacia el turismo, promovido tanto por actores externos al territorio como internos, incluyendo en esto a los pueblos indígenas que lo habitan. En: Francisca de la Maza, 2018, Tourism in indigenous territories: impact of public policies and the tourism value of indigenous culture. Journal Latin American and Caribbean Ethnic Studies. 13(1): 94-111. 